tado un sistema de remisión o derivación que trata de dirigir los escasos recursos - por ejemplo, los mejores profesionales y el equipo más avanzadoa las personas que más los necesitan. Según un estudio inicial, esta política ha logrado buenos resultados en zonas pobres de la Ciudad de Guatemala, donde las comadronas tradicionales trabajan en centros locales de maternidad bajo la supervisión de un médico y del personal de enfermería.

No obstante, un sistema basado en la derivación para responder a las emergencias obstétricas puede no tener éxito si no hay un médico que supervise el parto. A pesar de que el MSPAS ha tratado de aliviar este problema mediante el entrenamiento de comadronas cakchiqueles que atienden los partos en los hogares, no se puede esperar que estas abandonen sus costumbres y prácticas solo para cumplir con las directivas del MSPAS. Esas directivas deben adaptarse al sistema de conocimientos ya existente.

Aunque las directivas promueven las derivaciones preventivas y hacen un llamado a las mujeres a que acudan a los hospitales antes de que surjan complicaciones, no tienen en cuenta el deseo de las embarazadas de tener un parto vaginal normal en su hogar, rodeada de sus familiares. Por eso, la mayoría de las mujeres cakchiqueles preferirán esperar a que surja un problema para acudir al hospital, antes de sacrificar innecesariamente la rica experiencia de un parto en las condiciones que ellas desean. (Berry NS. Kaqchikel midwives, home births, and emergency obstetric referrals in Guatemala: contextualizing the choice to stay at home. Soc Sci Med. 2006;62(8):1958-69.)

\section{Percepciones acerca del uso de fármacos abortivos en mujeres de Colombia, Ecuador, México y Perú}

A pesar de que el aborto está restringido legalmente en casi todos los países de América Latina, los servicios dedicados a esa actividad están ampliamente disponibles, especialmente en las áreas urbanas. Los hay caros y seguros o baratos y más peligrosos. Se calcula que $29 \%$ de las mujeres en edad reproductiva se someten a abortos mediante procedimientos riesgosos, y las mujeres pobres o que viven en áreas rurales se encuentran aun en mayor desventaja. Según los códigos penales de Colombia, Ecuador, México y Perú, el aborto inducido es un crimen, salvo muy pocas excepciones. Se calcula que entre 1995 y 2000, alrededor de $28 \%$ de las muertes maternas en Colombia, $18 \%$ en Ecuador, $23 \%$ en México y $16 \%$ en Perú se debieron a abortos riesgosos. Aunque se han realizado pocos esfuerzos para mejorar el acceso a procedimientos más seguros, se han puesto en marcha programas para la atención de las complicaciones provocadas por los abortos.

Se realizaron entrevistas a profundidad a 49 mujeres entre 18 y 44 años de edad (mediana: 25 años) que habían consumido un fármaco abortivo bajo supervisón clínica en zonas rurales urbanas de Colombia, Ecuador, México y Perú, con la finalidad de conocer su percepción sobre el tema. La captación de estas mujeres para la investigación estuvo a cargo de los propios médicos que realizaron los abortos; las entrevistas se efectuaron entre octubre de 2003 y mayo de 2004.

Las participantes de los cuatro países prefirieron someterse al uso de un abortivo por ser menos doloroso, más sencillo, más natural, menos invasor y menos peligroso que el aborto quirúrgico. Por lo general lo describieron como un proceso natural de regulación menstrual. El hecho de que ofrecía mayor privacidad y era menos costoso también influyó en la decisión.

A excepción de las participantes de México, las mujeres encuestadas no estaban bien informadas acerca de la necesidad de emplear métodos anticonceptivos con regularidad, o no habían incorporado esa información adecuadamente a su forma de vida. Algunas entrevistadas afirmaron que sus parejas habían ofrecido tomar las precauciones para evitar el embarazo, pero que no lo habían cumplido.

De las 49 entrevistadas, $37(75,5 \%)$ tuvieron un aborto satisfactorio con el fármaco ingerido, mientras que $12(24,5 \%)$ recurrieron al aborto quirúrgico después del fracaso del abortivo. En total, 38 mujeres $(77,6 \%)$ usaron misoprostol —de ellas $30(78,9 \%)$ lograron un aborto satisfactorio- y $11(22,4 \%)$ usaron metotrexato con misoprostol, con lo cual obtuvieron buenos resultados en $7(63,6 \%)$ casos. Los embarazos tuvieron una duración mediana de 7 semanas.

Las mujeres que consideraban el aborto un pecado tuvieron mayores dificultades, mientras que las que comprendían los cambios que ocurrían en su organismo al abortar soportaron mejor el proceso. La participación de un médico y el apoyo de la pareja fueron factores clave para la estabilidad de las mujeres.

El uso de un abortivo goza de la aceptación de un amplio espectro de mujeres en lugares donde se prohíbe legalmente, por lo que se debe poner a su 
disposición. Si se toma en cuenta que el aborto con medicamentos no es necesariamente el método adecuado para todas las mujeres, se debe evitar que el costo sea el factor decisivo en la elección. El apoyo psicosocial que las mujeres reciben durante el proceso de aborto resulta crucial, especialmente si consideran que el aborto es un pecado, son jóvenes o pobres, no conocen bien su organismo, carecen del apoyo de su pareja o quedaron embarazadas como resultado de actos de violencia sexual. (Lafaurie MM et al. Women's perspectives on medical abortion in Mexico, Colombia, Ecuador and Peru: a qualitative study. Reprod Health Matters. 2005;13(26):75-83.)

\section{Abandono espontáneo del hábito de fumar durante el embarazo en mujeres estadounidenses de bajos ingresos}

Diversas investigaciones han asociado el hábito de fumar cigarrillos durante el embarazo con el peso bajo al nacer, el nacimiento prematuro, el aumento del riesgo de complicaciones durante el parto y el aborto espontáneo. Por ello es importante conocer los factores que favorecen que una mujer abandone de manera espontánea ese hábito y saber si ese abandono será prolongado.

En este estudio preliminar se comparan algunas características de las mujeres de bajos ingresos que abandonaron espontáneamente el hábito de fumar cigarrillos con las de mujeres que mantuvieron ese hábito. Participaron en él 141 mujeres embarazadas que fumaban cigarrillos o que habían abandonado ese hábito sin ayuda profesional al saber de su embarazo. Las participantes eran mayores de 18 años y tenían menos de 30 semanas de gestación. Las mujeres que abandonaron el hábito de fumar espontáneamente se dividieron aleatoriamente en dos grupos: uno recibió tratamiento especializado para evitar que se volviera a fumar y otro, el grupo testigo, recibió el tratamiento habitual.

En total, 33 mujeres (23\%) abandonaron espontáneamente el hábito de fumar cigarrillos. Los resultados demostraron que estas mujeres tenían más confianza en sí mismas, eran más jóvenes y fumaban menos que las que no dejaron de fumar. Cada una de estas variables tuvo una contribución independiente de $25 \%$ al abandono espontáneo del hábito de fumar.

Al final del período de gestación, $76 \%$ de las participantes habían dejado de fumar y $36 \%$ del total seguía sin fumar 6 meses después del parto. La psicoterapia no aportó ninguna protección adicional contra la recaída en el subgrupo que dejó de fumar espontáneamente.

Los datos derivados de esta investigación, junto con otros obtenidos en investigaciones anteriores, indican que si bien es baja la tasa de recaída inmediatamente después del parto en las mujeres que abandonaron espontáneamente el hábito de fumar cigarrillos, la mayoría de estas habían retomado ese hábito 6 meses después. Se deben reforzar las acciones dirigidas a evitar la recaída en el período posterior al parto.

Estos resultados se basan en entrevistas con mujeres que aceptaron participar voluntariamente en el estudio, del que formaba parte una intervención para dejar de fumar, por lo que no son representativos de todas las mujeres embarazadas que fuman. No obstante, dada la importancia que tiene para la salud pública el abandono de este hábito nocivo, especialmente en mujeres embarazadas, estos resultados pueden ayudar a los proveedores de salud a estructurar campañas antitabáquicas más eficaces dirigidas a este grupo de la población. (Morasco BJ et al. Spontaneous smoking cessation during pregnancy among ethnic minority women: a preliminary investigation. Addict Behav. 2006;31(2):203-10.)

\section{Riesgo de transmisión de la tripanosomiasis americana por triatominos en México}

La tripanosomiasis americana, una de las principales enfermedades transmitidas por insectos en América Latina, es endémica en varios estados de México. En ese país la seroprevalencia es de 1,5\% en donantes de sangre y asciende a $20 \%$ en la población de algunas regiones. Además, en los últimos años se han diagnosticado varios casos de la enfermedad.

En México se conocen 31 especies de triatominos que pueden ser vectores del agente causal de la enfermedad y 18 de ellos están infectados de forma natural con el parásito Trypanosoma cruzi. La mayoría de las especies encontradas en las viviendas y en sus alrededores son de origen selvático.

Para caracterizar el nivel de infestación doméstica y peridoméstica con triatominos se estudiaron tres comunidades rurales de México: Felipe Carrillo Puerto (Estado de Nayarit), Cuxpala (Zacatecas) y Tepehuaje de Morelos (Jalisco) entre abril de 1998 y noviembre de 1999. Estas comunidades están ubicadas en regiones semiáridas, tienen condiciones atmosféricas similares y nunca han for- 